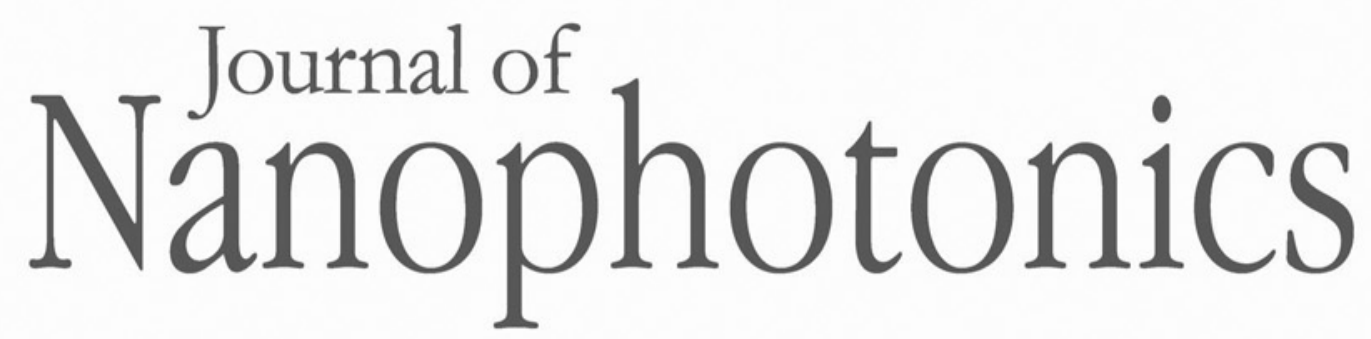

\title{
Flat focusing in reflection from a chirped dielectric mirror with a defect layer
}

Yu-Chieh Cheng

Simonas Kicas

Kestutis Staliunas 


\title{
Flat focusing in reflection from a chirped dielectric mirror with a defect layer
}

\author{
Yu-Chieh Cheng, ${ }^{\text {a,* }}$ Simonas Kicas, ${ }^{\text {b }}$ and Kestutis Staliunas ${ }^{\text {a,c }}$ \\ ${ }^{a}$ Universitat Politècnica de Catalunya, Departament de Física i Enginyeria Nuclear, \\ Rambla Sant Nebridi 22, Terrassa, Barcelona 08222, Spain \\ ${ }^{\mathrm{b}}$ State Research Institute for Physical Sciences and Technology, Savanoriu pr. 231, Vilnius \\ 02300, Lithuania \\ 'Institució Catalana de Recerca i Estudis Avançats (ICREA), Pg. Lluis Companys, 23, Barcelona \\ 08010, Spain
}

\begin{abstract}
Recently, the principle of flat focusing based on one-dimensionally chirped dielectric mirrors has been proposed and experimentally demonstrated. The flat chirped mirror causes anomalous diffraction of the beam during reflection, opposite to the normal diffraction in free space propagation. The anomalous diffraction compensates the normal diffraction of the beam resulting in focusing after reflection. For a better focusing performance and for a larger near-field focal distance, a stronger anomalous diffraction is required. We show that the anomalous diffraction can be enhanced by introducing a defect layer in the chirped mirror, as the structure becomes similar to a Gires-Tournois interferometer. The focal distance can be substantially increased due to the defect layer. In our specific structure, the focal distance shows an increase from 19 to $39 \mu \mathrm{m}$, numerically. (C) 2015 Society of Photo-Optical Instrumentation Engineers (SPIE) [DOI: 10.1117/1.JNP.9.093084]
\end{abstract}

Keywords: chirped dielectric mirror; Gires-Tournois interferometer; flat focusing mirror.

Paper 14121 received Oct. 17, 2014; accepted for publication Feb. 2, 2015; published online Mar. 4, 2015.

\section{Introduction}

One of the important properties of photonic crystals, apart from their well-known frequency bandgaps, ${ }^{1,2}$ is their anomalous refraction/diffraction. The latter property allows designing a flat focusing lens for near-field focusing/imaging of transmitted beams. ${ }^{3}$ One interesting peculiarity of the flat focusing lens is its transversal invariance, i.e., the lack of an optical axis. Such a transversally invariant lens with a flat interface had been first realized in the microwave frequency range ${ }^{3,4}$ but recently also in the visible range. ${ }^{5}$ This kind of flat lensing has also been demonstrated in acoustics by sonic crystals. ${ }^{6}$ The flat lens focusing is a near-field focusing and is principally different from the far-field focusing by conventional spherical or Fresnel lenses. For instance, the focal length $F$ of the near-field focusing follows the relation $F=l_{\text {object }}+l_{\text {image }}$ where $l_{\text {object }}$ and $l_{\text {image }}$ are the distances from a lens to an object and to an image, respectively. In contrast to a near-field lens, the well-known relation of a far-field lens is $1 / F=$ $1 / l_{\text {object }}+1 / l_{\text {image }}$, as follows geometrical optics. The limitation of near-field focusing is that the source and the image are usually close to the surface of the flat lens, and may only be a few micrometers apart, so a near-field focusing device with a long focal distance is challenging. In this way, the challenges in the near-field focusing and the far-field focusing are to increase or decrease the focal length $F$, respectively.

Recently, near-field focusing has also been proposed in reflections from flat mirrors. A beam was shown to focus in the reflection from a one-dimensional (1-D) chirped dielectric mirror, ${ }^{7}$ or from an optimized 1-D dielectric structure. ${ }^{8}$ By optimizing a chirped mirror, the focal length can be increased from a few tens to hundreds of micrometers. The flat mirror effect has also been

*Address all correspondence to: Yu-Chieh Cheng, E-mail: yu.chieh.cheng@upc.edu

1934-2608/2015/\$25.00 (C) 2015 SPIE 
demonstrated in reflections from two-dimensionally modulated (2-D) sub-wavelength dielectric gratings. ${ }^{9,10}$ There are, in fact, other arrangements for focusing a beam in reflection with engineered grating structures. ${ }^{11,12}$ However, the latter cases pose the optical axes and the lateral invariant is absent.

The principle of flat focusing mirrors ${ }^{7-10}$ also relies on anomalous diffraction, similar to the principle of flat lensing in transmission. A beam diverges during propagation in homogeneous dispersive media because of phase delays among different angular field components. The effective diffractive propagation length can be considered as the second derivative of the phase $\phi$ in an angular space, $L_{\mathrm{diffr}}=-k \cdot d^{2} \phi / d k_{x}^{2}$ where $k_{x}$ is the wave vector component in the transversal direction $\vec{k}=\left(k_{x}, k_{z}\right)^{7}$ Therefore, if the beam experiences an anomalous phase delay during reflection opposite to the phase delay that occurred during propagation in free space, the beam will focus at some point where the normal and anomalous diffractions are compensated. In this way, the focal length of the flat mirror can be calculated as $F=-L_{\mathrm{diffr}}=k \cdot d^{2} \phi / d k_{x}^{2}$, where $\varphi$ is the phase shift of the angular component in reflection.

The focal length $F$ or, respectively, the anomalous diffraction length $-L_{\text {diffr }}$ should be large enough to observe the effect in measurements, both in transmission through the flat lenses or in reflection from flat mirrors. For instance, focusing cannot be achieved if $F<l_{\text {object }}$ because the anomalous diffraction undercompensates the phase delay accumulated during normal diffraction. Another important ingredient for effective focusing is the angular bandwidth of the anomalous diffraction which determines the numerical aperture (NA) of a flat focusing lens or mirror. Generally, the broader the angular focusing range and the larger the value of the anomalous angular dispersion, the better focusing performance can be expected.

In this paper, a chirped Bragg mirror composed of dielectric Bragg mirrors with the local periods (layer thickness) varying along the mirror structure is proposed to provide the anomalous angular dispersion to observe the near-field focusing effect in reflection. It is well known that the chirped mirrors are used to compensate the chromatic dispersion of ultra-short pulses. ${ }^{13}$ In the case of positively (negatively) chirped mirrors, the wave components at larger frequencies penetrate deeper

(a)

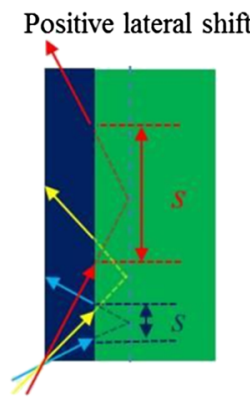

Anomalous lateral shift

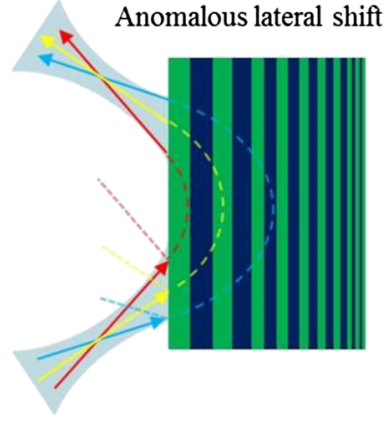

(b) Defect layer

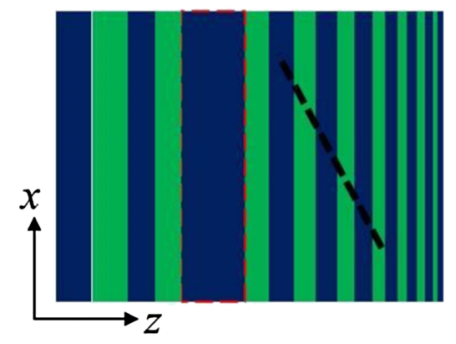

(c) Position of the defect layer

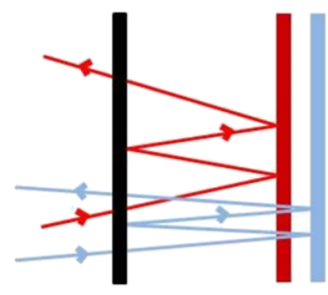

Fig. 1 Illustrations of the back mirror of a Gires-Tournois interferometer (GTI) structure substituted for chirped mirror. (a) A linearly chirped mirror allows the angular components at a smaller angle to penetrate deeper, which causes the larger lateral shift. A focusing or imaging effect can be observed. The dashed line is the beam reflecting from the metallic mirror as a reference. (b) A defect layer introduced at the fifth layer in the chirped mirror is framed with the red dashed line. (c) The defect in the chirped mirror can be considered as a GTI structure for different angular components. 
(shallower) into the structure during reflection. The penetration depth or group delay dispersion is frequency dependent, so it allows for compensating the chromatic dispersion of a pulse.

The idea of a flat focusing mirror for a narrow monochromatic beam is similar to the principle of compensating chromatic dispersion for a dispersive pulse. Figure 1(a) illustrates that normally, the propagation of each plane wave components of a beam follows Snell's law, and thus experiences zero or positive lateral shift. A beam reflecting from such an interface spreads because the lateral shift is proportional to the incidence angles, as illustrated by the dashed lines in Fig. 1(a). Next, a positively chirped mirror is introduced to obtain the opposite situation. The plane wave component at a larger incidence angle penetrates shallower than the components at a smaller angle which means that the plane wave component at a larger incidence angle experiences a smaller lateral shift. This effect leads to the anomalous slope of the lateral shift on the incidence angles which eventually can modulate or suppress the normal diffraction to obtain focus or image in reflection as indicated in Fig. 1(a) with solid lines.

\section{Idea of One-Dimensional Chirped Mirror with a Defect Layer}

Flat focusing in a chirped mirror was first demonstrated in Ref. 7. The angular dependence of the phase shift, however, was not monotonic as was initially expected. For instance, the slope of lateral shift on the incidence angles is not anomalous for all ranges of incidence angles, but is separated by areas with normal slopes. The peaks of negative diffraction appear as the slope changes from positive to negative and they result in fringes of dispersion curves. These fringes correspond to high values of anomalous diffractions in narrow angular ranges. Several solutions to eliminate the oscillations such as double-chirping ${ }^{14}$ and anti-reflection coating ${ }^{15}$ have been proposed. Although a broader and smoother dispersion can be obtained by partially eliminating these fringes of dispersion, the magnitudes of anomalous diffractions are simultaneously reduced. This makes the focusing performance even worse. On the other hand, the several peaks of anomalous diffraction can even enhance the focusing performance. One possibility for enhancing the peaks of anomalous diffractions is to introduce a defect into the structure as depicted in Fig. 1(b). For instance, a Gires-Tournois interferometer (GTI) is composed of a defect layer in between two mirrors: the front mirror is partially reflective and the back mirror is highly reflective. ${ }^{16}$ This could introduce a large, narrow peak of anomalous dispersion in chromatic or angular spectra. The large anomalous dispersion appears in a very narrow spectral (angular) range; therefore, focusing is practically absent for a simple GTI structure. On the other hand, a chirped mirror with a defect layer is proposed in this paper to provide a larger anomalous diffraction in a broader angular bandwidth to give a larger focal length.

In order to manipulate fringes, the analysis of the fringes is first explored. The condition of the fringes is $k_{z} \cdot 2 l=2 \pi m(m=1,2,3 \ldots)$ where $k_{z}$ is the longitudinal component of the wave vector and $l$ is the linear cavity length. This condition is, however, valid when both mirrors are similar, i.e., both do or both do not impose the $\pi$ phase shift of the reflecting wave. This happens when the defect layer (serving the role of mirror) is the layer with a low refractive index. On the other hand, if the defect layer is the layer with a high index, a $\pi$ shift occurs, which results in the modification of the resonance condition $m^{\prime}=m-1 / 2(m \prime=0.5,1.5,2.5 \ldots)$. When the defect layer is introduced into the chirped mirror, the cavity length $l$ can be estimated to be the distance between the defect layers and the reflecting point of the chirped mirror. Therefore, we simply obtain the relation between the resonant incidence angle $\alpha$ and the cavity length $l$.

$$
\frac{1}{\cos (\alpha)}=\frac{2 l}{\lambda m} \text {. }
$$

\section{Numerical Calculations}

The chirped mirror in our calculations is composed of layers of alternating high and low refractive index materials with $n_{\mathrm{H}}=2.17$ and $n_{\mathrm{L}}=1.49$ for the wavelength $\lambda=532 \mathrm{~nm}$, just as the structure considered in Ref. 7. The thickness of the defect layer is supposed to be double that of the original layer. Figure 2(a) shows the numerical focal length, calculated as the second-order 
(a)

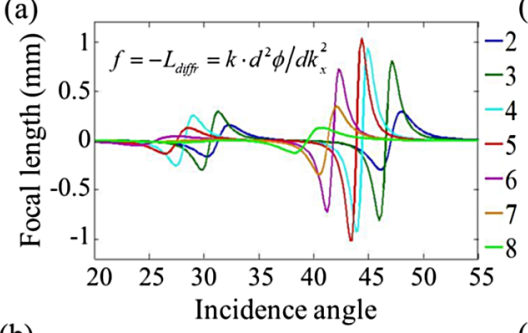

(b)

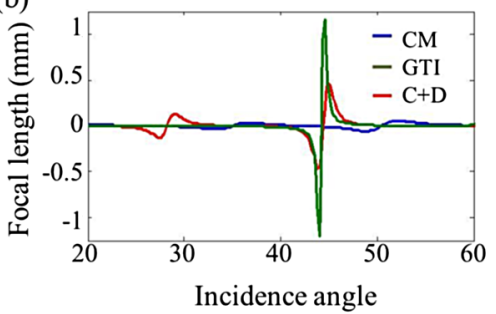

(c)

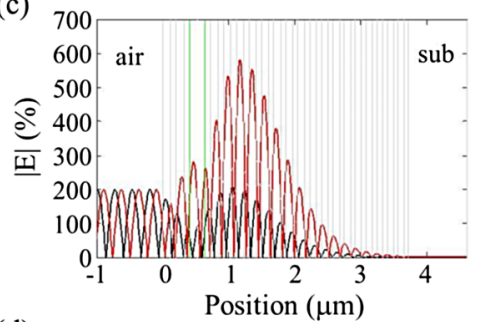

(d)

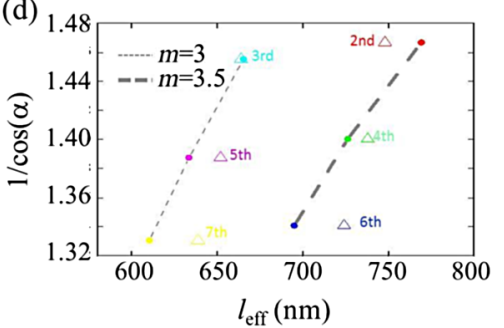

Fig. 2 Properties of the chirped mirror with a defect. (a) The focal length depends on different incidence angles for different locations of defect layer from the second to eighth layers. The second to eighth layer thicknesses of the chirped mirror are $88,116,87,115,86,113$, and $85 \mathrm{~nm}$. The defect layers are the double thicknesses of their local layers. (b) The comparison of the focal length between the chirped mirrors (blue), GTI structure (green), and the chirped mirror with a defect located at the fifth layer (red). (c) The inner field distribution at 44.37 deg as the defect layer is placed at the fifth layer with a thickness of $230 \mathrm{~nm}$ (green lines). The red and black lines show the electric fields of the chirped structure with and without a defect layer. (d) The effective cavity length depends on the incidence angle $\alpha$. Triangles represent the calculations' results. The dots show the expectation from Eq. (1). The two dashed lines show different mode numbers.

derivative of the phase of the refractive coefficient with respect to incidence angles, for different positions of the defect from the second to eighth layers of the chirped mirror structure.

The largest numerical focal length was obtained when the defect was positioned on the fifth layer in our specific case where the reflectance of the front mirror of GTI is optimal. The defect layer can be regarded as a low reflecting front mirror of GTI and the chirped mirror plays the role of a highly reflective back mirror of GTI. Importantly, after introducing a defect layer in the chirped mirror, the angular ranges for the positive focal length remain nearly the same, but the value of the diffractive length is largely enhanced as shown in Fig. 2(b). It is also noted that the angular variance of this proposed structure is broader than the one of a simple GTI structure (green line) because the layers behind the defect layer act as a chirped mirror reflecting a broad range of angular components.

It is observed that the peaks of the focal length shift to the small angles as the defect layer is introduced at deeper layers. This can be simply explained from Eq. (1) where the cavity length $l$ is proportional to the resonant angles $\alpha$. The cavity length $l$ can be calculated by the distance between the defect layer and the reflecting point. The reflecting point cannot be precisely found and it is roughly estimated to coincide with the position of the maximum of the field.

The inner field distribution can indicate the position of the reflecting point. For example, Fig. 2(c) shows the inner field distribution at resonant angle 44.37 deg defined at the maximum value of the fringe [the red line in Fig. 2(a)] with and without the defect layer as shown with red and black lines. The reflecting point is around the 11th layer where the higher electric envelope appears. The effective cavity length can be calculated between the reflecting point (11th layer) and the defect layer (5th layer). Here, it is assumed that the front mirror is at the defect layer (the 5th layer) because we notice the electric field in the defect layer [red line in Fig. 2(c)] is slightly enhanced. It is assumed that the field reflects between the defect layer (the lower electric envelope) and the reflecting point (the higher electric envelope). On the other hand, the electric field from the chirped mirror without a defect layer [black line in Fig. 2(c)] shows only an envelope at the 10th layer where the reflecting point is. The effective cavity length $l_{\text {eff }}$ is calculated around $652 \mathrm{~nm}$ and the cavity length $l$ calculated by Eq. (1) is $634 \mathrm{~nm}$ at $44.37 \mathrm{deg}$, marked as the purple dot in Fig. 2(d). There is only an $18 \mathrm{~nm}$ difference. Although these values are not 


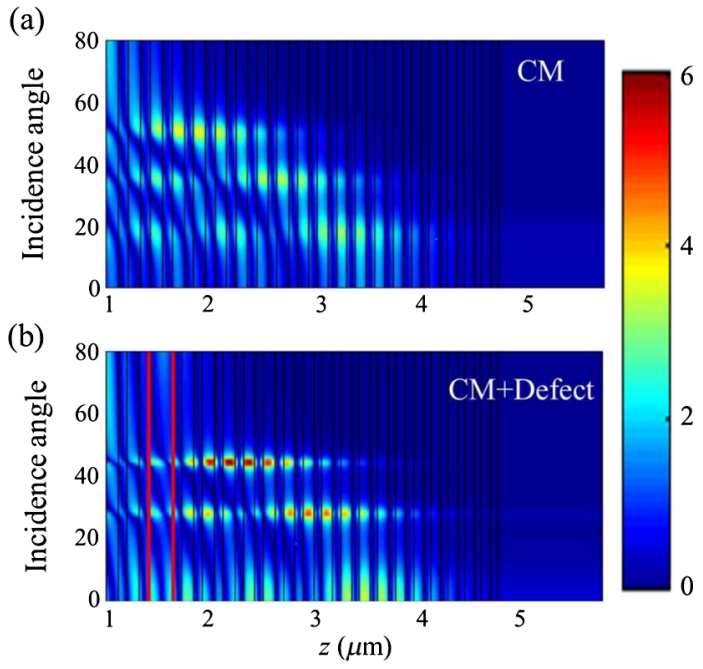

Fig. 3 The two-dimensional (2-D) map of field inside the mirror structures depending on the incidence angle for (a) the chirped mirror and (b) the chirped mirror with a defect at the fifth layer. The interface between air and the structure is at $z=1 \mu \mathrm{m}$. Each black line indicates the boundary of each layer. The position of the defect layer is marked in red lines.

exactly the same, they are still in agreement with the expected tendency from Eq. (1). The variation may be mainly caused by the definition of incidence angles from Fig. 2(a). For example, with a slightly different incidence angle of $2 \mathrm{deg}$, the maximum peak of inner field can shift over $50 \mathrm{~nm}$.

Figure 2(d) also shows another case as the defect layer is introduced at the chirped mirror from the second to the seventh layers. The dashed line is calculated from Eq. (1) as the mode number $m$ is supposed to be $m=3$ or $m=3.5$ depending on whether the defect layer has a low/ high refractive index. The tendency of the effective cavity length matches the expectation from Eq. (1) with a variation of $\pm 30 \mathrm{~nm}$. It is also proven that the effective cavity length is from the defect layer (front mirror) to the higher electric envelope (back mirror) just as we proposed.

Figure 3 shows the field distribution inside the structure depending on the incidence angles. It shows that: (1) the smaller incidence angles can penetrate deeper and the larger one reflect in advance. (2) The incidence angles of the inner field peaks in Fig. 3 correspond to the ones of the focal length peaks in Fig. 2(b). For instance, both the peaks of the focal length and the field enhancement in the defect case appear at incidence angles of $29 \mathrm{deg}$ and $45 \mathrm{deg}$. The stronger focal length at $45 \mathrm{deg}$ contributes a stronger field enhancement. (3) The effective cavity length $l_{\text {eff }}$ of a chirped mirror with a defect is reduced so all patterns shift to smaller angles as expected.

\section{Focusing Performance}

The focal length cannot be directly obtained from the equation $F=-L_{\mathrm{diffr}}=k \cdot d^{2} \phi / d k_{x}^{2}$ which is used in Fig. 2(b). This is because the different plane wave components of the beam located inside the positive peak of the focal length in Fig. 2(b) experience different negative diffraction lengths. The peak value of the numerical focal length does not represent the focusing distance from the mirror. Nevertheless, it can indicate the focusing angle at $45 \mathrm{deg}$ where the slope of the lateral shift (the first derivation) of angles is negative or the numerical focal length (the secondary derivation) is positive. It should be also noted that when the incident angle is slightly smaller or larger than the focusing angle, the plane wave components of the beam simultaneously experience normal and anomalous diffractions which results in beam splitting. Figure 4(b) shows some indication of beam splitting, however, not all of the plane wave components of the beam are located inside the positive peak of the focal length [Fig. 2(b)].

Figure 4 shows that the focusing performance is calculated by the paraxial model at the incidence angle $\alpha=45 \mathrm{deg}$. Figures 4(a) and 4(b) show the focusing effect after introducing 

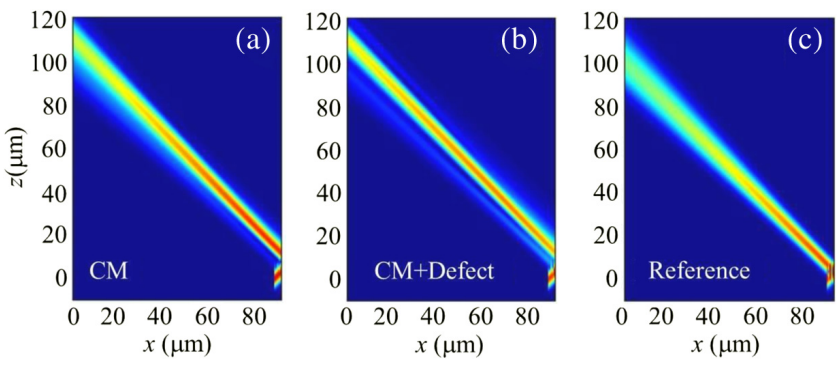

Fig. 4 The performance of three flat mirrors. The beam propagation in reflection for (a) chirped mirror, (b) chirped mirror with a defect, and (c) the metallic mirror (as a reference beam) at the angle $\alpha=45$ deg for TE polarization.

the defect in the chirped mirror. The diverging reflected beam from the conventional metallic mirror is shown in Fig. 4(c). The source is positioned at $l_{\text {object }}=5 \mu \mathrm{m}$ in front of the mirror and the width of the source beam is $8 \mu \mathrm{m}$. The wavelength of the source is $\lambda=532 \mathrm{~nm}$ and transverse electric (TE) polarization (Ey) characterized by its electric field being perpendicular to the plane of incidence (xz-plane) is considered.

The focal distance in reflection can be increased from $l_{\text {image }}=14 \mu \mathrm{m}$ to $l_{\text {image }}=34 \mu \mathrm{m}$ as expected as the source is fixed at $l_{\text {object }}=5 \mu \mathrm{m}$. The focal length $F$ of near-field focusing $F=$ $l_{\text {image }}+l_{\text {object }}$ is increased from 19 to $39 \mu \mathrm{m}$. We note that the focal distance $F=39 \mu \mathrm{m}$ is within the near-field area, as the distance is smaller than the Rayleigh range of our beam source, $Z_{r}=W^{2} / \lambda=8^{2} / 0.532=120 \mu \mathrm{m}$.

\section{Conclusion}

In conclusion, we demonstrate that a defect, introduced at the position close to the front face of the chirped mirror, can act as a front mirror of the GTI structure. An enhancement of the fringes of angular dispersion is obtained due to the defect layer, which increases the focal length in reflection from a flat focusing mirror, whereas the angular range of the focal length $F$ remains unaltered. In our specific calculations, the focal length $F$ increased from 19 to $39 \mu \mathrm{m}$ due to the defect layer in the chirped mirror structure.

\section{Acknowledgments}

We acknowledge financial support by the Spanish Ministerio de Educación y Ciencia and European FEDER (Project No. FIS2011-29734-C02-01), and by the European Union Structural Funds project. Y. C. Cheng was supported by the Erasmus Mundus Doctorate Program Europhotonics 2011/2014.

\section{References}

1. S. John, "Strong localization of photons in certain disordered dielectric superlattices," Phys. Rev. Lett. 58, 2486 (1987).

2. E. Yablonovitch, "Inhibited spontaneous emission in solid-state physics and electronics," Phys. Rev. Lett. 58, 2059 (1987).

3. P. V. Parimi et al., "Photonic crystals: imaging by flat lens using anomalous refraction," Nature 426, 404 (2003).

4. A. A. Houck, J. B. Brock, and I. L. Chuang, "Experimental observations of a left-handed material that obeys Snell's law," Phys. Rev. Lett. 90, 13740 (2003).

5. L. Maigyte et al., "Flat lensing in the visible frequency range by woodpile photonic crystals," Opt. Lett. 38, 2376 (2013).

6. V. J. Sánchez-Morcillo et al., "Propagation of sound beams behind sonic crystals," Phys. Rev. B 80, 134303 (2009).

7. Y. C. Cheng et al., "Beam focusing in reflection from flat chirped mirror," Phys. Rev. A 87, 045802 (2013). 
8. Y. C. Cheng et al., "Flat focusing mirror," Sci. Reports 4, 6326 (2014).

9. Y. C. Cheng, J. Redondo, and K. Staliunas, "Beam focusing in reflections from flat subwavelength diffraction gratings," Phys. Rev. A 89, 033814 (2014).

10. Y. C. Cheng et al., "Beam focalization in reflection from flat dielectric subwavelength gratings," Opt. Lett. 39, 6086 (2014).

11. L. Verslegers et al., "Planar lenses based on nanoscale slit arrays in a metallic film," Nano Lett. 9, 235 (2009).

12. D. Fattal et al., "Flat dielectric grating reflectors with focusing abilities," Nat. Photonics 4, 466 (2010).

13. R. Szipöcs and A. Koházi-Kis, "Theory and design of chirped dielectric laser mirrors," Appl. Phys. B 65, 115 (1997).

14. N. Matuschek et al., "Back-side-coated chirped mirrors with ultra-smooth broadband dispersion characteristics," Appl. Phys. B 71, 509 (2000).

15. F. Gires and P. Tournois, "Interferometre utilisable pour la compression d'impulsions lumineuses modulees en frequence," C. R. Hebd. Acad. Sci. Paris 258, 6112 (1964).

16. L. A. A. Pettersson, L. S. Roman, and O. Ingana, "Modeling photocurrent action spectra of photovoltaic devices based on organic thin films," J. Appl. Phys. 86, 487 (1999).

Yu-Chieh Cheng is a joint PhD student at the Universitat Politecnica de Catalunya (UPC), Barcelona, Spain, and the European Laboratory for Non-Linear Spectroscopy (LENS), Florence, Italy. She received her BD and MD degrees from National Central University, Taiwan, and continues her $\mathrm{PhD}$ in Europe, sponsored by the EuroPhotonics program of Erasmus Mundus Joint Doctorate (EMJD). Her PhD study is on photonic crystals (PhCs) for beam modulations and resonant cavities. Presently, she has published 8 journal papers.

Simonas Kicas is a PhD student at the Center for Physical Science and Technology (CPST), Vilnius, Lithuania. He received his BD and MD degrees from Vilnius University (Lithuania) and continues his $\mathrm{PhD}$ in the field of thin-film coating deposition technologies and dispersive multilayer systems. To date, he has published 5 journal papers.

Kestutis Staliunas is a full-time professor of ICREA at Universitat Politecnica de Catalunya (UPC), Barcelona, Spain. He received his PhD degree in physics from Vilnius University (Lithuania) in 1989. He is the author of more than 200 journal papers and of a monograph (K. Staliunas, and V. J. Sanchez-Morcillo, "Transverse Patterns in Nonlinear Optical Resonators," Springer, 2003). His current research interests include light-pattern formation in nonlinear optical systems, photonic crystals, metamaterials, and others. 